\title{
Diagnostic delay of pulmonary nontuberculous mycobacterial infection in China
}

\author{
Hui Jing ${ }^{1 \dagger}$, Wanming Tan ${ }^{1 \dagger}$, Yunfeng Deng ${ }^{1}$, Dachuan Gao ${ }^{1}$, Liang Li' ${ }^{2}$ Zhiming Lu ${ }^{3}$, Edward A Graviss ${ }^{4}$ \\ and Xin $\mathrm{Ma}^{1,4^{*}}$
}

\begin{abstract}
Background: Nontuberculous mycobacteria (NTM) infection is an emerging, but neglected public health concern in China.

Findings: To investigate diagnostic delay of NTM diseases in China, we analyzed 91 patients with pulmonary NTM infection in ShandongProvince. The median diagnostic delay time of the analyzed patients was 84 days, which was significantly associated with rural inhabitance ( 135 days vs. 73 days of urban inhabitance, $p<0.01$ ) and lower level of first visiting hospitals/clinics (70 and 82 days of tertiary and secondary hospitals/clinics respectively vs. 120 days of primary hospitals/clinics, $\mathrm{p}<0.05)$. M. farcinogenes was isolated from a 79-year-old male patient, which is the first report of pulmonary infection in humans.
\end{abstract}

Conclusions: Our results indicate a significant diagnostic delay of NTM diseases in China, especially for rural patients with limited access to higher-level healthcare services.

Keywords: China, Infection, Nontuberculous mycobacterium

\section{Introduction}

Nontuberculous mycobacteria (NTM) diseases have been neglected in most of developing world [1]. With the global pandemic of HIV/AIDS and aging population, NTM have been increasingly associated with pulmonary diseases in humans [2]. In China, Mycobacterium tuberculosis (MTB) still causes the majority of pulmonary mycobacteria diseases. Nevertheless, in the last decade, the NTM isolation rate has shown an increasing trend in China [3,4]. Pulmonary NTM diseases may share clinical signs with $\mathrm{TB}$, causing a clinical dilemma in early diagnosis and treatment. Delay or misdiagnosis of NTM diseases may worsen the disease and increase the mortality and economic burden of patients. In this study, we retrospectively investigated the diagnostic history and clinical characteristics of patients with pulmonary NTM

\footnotetext{
* Correspondence: mx79@hotmail.com

${ }^{\dagger}$ Equal contributors

'Katharine Hsu International Research Center of Human Infectious Diseases, Shandong Provincial Chest Hospital, Shandong University, Jinan, Shandong 25013, China

${ }^{4}$ The Center for Molecular and Translational Human Infectious Diseases Research, Houston Methodist Hospital Research Institute, Houston, Texas 77030, USA

Full list of author information is available at the end of the article
}

diseases to better understand the factors related to the diagnostic delay in China.

\section{Study population and methods}

This study was conducted in Shandong Province, which is the second largest province in China (population size, 96 million and 59\% rural) and has approximately 40,000 new TB cases annually. Shandong Provincial Chest Hospital $(\mathrm{SPCH})$ is the only provincial-level hospital specialized in TB (a referral hospital) and other lung infections, and networks 139 county/city-level TB clinics. Between January 1, 2007 and December 31, 2012, 91 patients were diagnosed with pulmonary NTM disease based on the American Thoracic Society/Infectious Disease Society of America (ATS) diagnostic criteria [5]. Multiple $(\geq=2)$ sputum specimens from each patient were collected for Acid Fast Bacillus (AFB) test and mycobacterial culture at SPCH. Identification of mycobacteria species was first carried out at the ISO15189-certified SPCH TB reference laboratory by conventional biochemical tests, P-nitrobenzoic acid (PNB) and 2-Thiophene carboxylic acid hydrazide (TCH) testing following a standard protocol [6], and further 
identified by $16 \mathrm{~S}$ rRNA gene sequence analysis (MicroSeq ID Microbial Indentification Software V2.0, PE Applied Biosystems) at the species level as previously described [7]. To minimize possible species identification error, DNA extraction and $16 \mathrm{~S}$ rRNA gene sequencing of each NTM isolate was independently conducted twice. Statistical analysis was carried out by using SPSS (IBM, USA). The study protocol was approved by the SPCH Institutional Review Board.

\section{Results}

Over the six study years, a total of 91 patients (mean age $\pm \mathrm{SD}, 45.7 \pm 16.2$ years; age range, 15 - 79 years; male, 73.6\%) were identified with pulmonary NTM infection from 4541 patients with positive mycobacteria cultures at $\mathrm{SPCH}$, indicating an overall NTM isolation rate of $2.0 \%$ among all mycobacterial isolates, that did not show a significantly increasing or decreasing trend within the study period. The patients were predominantly male $(73.6 \%)$ and adult (98.9\% older than 18 years; and $37.4 \%$ from age group of $46-64$ years) (Table 1 ). The diagnostic delay of NTM patients was defined as the period from the date of the patient's first visit at the healthcare service to the date of diagnosis. Median diagnostic delay time for the 91 NTM patients was 84 days (range, 28 - 1,144 days), and it was not significantly associated with sex and age of NTM patients (Table 1). Patients living in rural areas showed a significantly longer diagnostic delay time than those in the urban areas

\begin{tabular}{|c|c|c|c|}
\hline Patient characteristics & $\begin{array}{l}\text { N. (\%) } \\
(\text { N. = 91) }\end{array}$ & $\begin{array}{l}\text { Median diagnostic } \\
\text { delay (Days) }\end{array}$ & $\mathbf{p}$ \\
\hline \multicolumn{4}{|l|}{ Sex } \\
\hline Male & 67 (73.6) & 86 & \\
\hline Female & $24(26.4)$ & 77 & 0.86 \\
\hline \multicolumn{4}{|l|}{ Age (years) } \\
\hline $1-17$ & $1(1.1)$ & 101 & \\
\hline $18-25$ & $19(20.9)$ & 66 & \\
\hline $26-45$ & $23(25.3)$ & 81 & \\
\hline $46-65$ & $34(37.4)$ & 91 & \\
\hline$>65$ & $14(15.3)$ & 99 & 0.71 \\
\hline \multicolumn{4}{|l|}{ Inhabited areas } \\
\hline Urban & $61(67.0)$ & 73 & \\
\hline Rural & $30(33.0)$ & 135 & $<0.01$ \\
\hline \multicolumn{4}{|c|}{ First visiting hospitals/clinics } \\
\hline Tertiary & $45(49.5)$ & 70 & \\
\hline Secondary & $20(22.0)$ & 82 & \\
\hline Primary & $26(28.5)$ & 120 & $<0.05$ \\
\hline
\end{tabular}

(135 vs. 73 days, $\mathrm{p}<0.01$ ). Patients with a first visit at tertiary and secondary hospitals/clinics had significantly shorter delay time than those at primary hospitals/clinics (70 and 82 days respectively vs. 120 days, $\mathrm{p}<0.05$ ) (Table 1).

All 91 NTM patients were HIV seronegative. Other comorbidities included diabetes (9.9\%), cardiovascular diseases (13.2\%), chronic obstructive pulmonary disease (COPD, 12.1\%), and bronchiectasis (20.9\%) (Table 2). Mycobacterium (M.) intracellulare infection accounted for $46.1 \%$ of these NTM diseases, following by $M$. chelonaeabscessus complex (28.6\%), M. kansasii (12.1\%), M. gordonae (5.5\%), M. fortuitum (5.5\%), M. asiaticum (1.1\%), and M. farcinogenes (1.1\%). In this study, a 79-year-old male patient was diagnosed with severe COPD, bronchiectasis, and pulmonary infection Positive results were shown in six consecutive sputum AFB smear tests. Mycobacterial culture and identification revealed isolation of $M$. farcinogenes. It is the second report of human infection and the first report of pulmonary $M$. farcinogenes disease in humans [8].

\section{Discussion}

Our data suggest that the diagnosis of pulmonary NTM disease is significantly delayed in China, which is attributed to rural inhabitance, and limited access to higher-level healthcare and laboratory services (tertiary and secondary). In China, approximately $71.3 \%$ of patients with active TB live in the resource-poor rural areas, where the mycobacteria culture and species identification tests are not routine testing for each TB or NTM suspect yet [8]. In our previous studies in Shandong, without species identification testing, NTM disease was often misdiagnosed as multidrug resistant TB (MDR-TB) and accounted for 30.7\% of reported MDR-TB (due to natural resistance of NTM to anti-TB drugs) [4]. Approximately 4\% of retreated TB cases were caused by NTM infections [4]. The 2010 Chinese National TB Surveillance Program has reported a NTM isolation rate of $22.9 \%$ among 363 culture positive TB suspects [9]. This data strongly indicates that NTM infection has become a significant public health concern in China, which should not be neglected in disease control practice. The clinicians and public health professionals in primary care settings need to improve their awareness of this emerging disease. Mycobacteria culture and species identification tests need to be routine testing for each TB or NTM suspect.

This study is a hospital-based surveillance study with a limited sample size, which aims to investigate the diagnostic delay caused by the healthcare system. There are definitely some other factors, such as patients' comorbid diseases and environmental risks [10], also contributing to the delay of care, which need to be investigated in future studies. 
Table 2 Clinical characteristics of patients infected with different NTM species

\begin{tabular}{|c|c|c|c|c|c|c|c|c|}
\hline & M. intracellulare & M. chelonae/abscessus complex & M. kansasii & M. gordonae & M. fortuitum & M. asiaticum & M. farcinogenes & Total \\
\hline $\mathrm{N}(\%)$ & $42(46.1)$ & $26(28.6)$ & $11(12.1)$ & $5(5.5)$ & $5(5.5)$ & $1(1.1)$ & $1(1.1)$ & $91(100.0)$ \\
\hline \multicolumn{9}{|l|}{ Symptoms } \\
\hline Chest pain/distress & $10(23.8)$ & $14(53.8)$ & $1(9.1)$ & $0(0.0)$ & $2(40.0)$ & $1(100.0)$ & $0(0.0)$ & $28(30.8)$ \\
\hline Cough & $36(85.7)$ & $18(69.2)$ & $9(81.8)$ & $4(80.0)$ & $4(80.0)$ & $0(0.0)$ & $1(100.0)$ & $72(79.1)$ \\
\hline Hemoptysis & $8(19.0)$ & $6(23.1)$ & $6(54.5)$ & $2(40.0)$ & $1(20.0)$ & $0(0.0)$ & $0(0.0)$ & $23(25.3)$ \\
\hline Fever/Sweats & $21(50.0)$ & $16(61.5)$ & $1(9.1)$ & $1(20.0)$ & $1(20.0)$ & $0(0.0)$ & $1(100.0)$ & $41(45.1)$ \\
\hline \multicolumn{9}{|l|}{ Comorbidities } \\
\hline \multicolumn{9}{|l|}{ Comorbid conditions } \\
\hline Diabetes & $4(9.5)$ & $2(7.7)$ & $1(9.1)$ & $1(20.0)$ & $1(20.0)$ & $0(0.0)$ & $0(0.0)$ & $9(9.9)$ \\
\hline Cardiac disease & $6(14.3)$ & $0(0.0)$ & $2(18.2)$ & $2(40.0)$ & $2(40.0)$ & $0(0.0)$ & $0(0.0)$ & $12(13.2)$ \\
\hline COPD & $8(19.0)$ & $2(7.7)$ & $0(0.0)$ & $0(0.0)$ & $0(0.0)$ & $0(0.0)$ & $1(100.0)$ & $11(12.1)$ \\
\hline Bronchiectasis & $11(26.2)$ & $5(19.2)$ & $0(0.0)$ & $0(0.0)$ & $1(20.0)$ & $1(100.0)$ & $1(100.0)$ & $19(20.9)$ \\
\hline \multicolumn{9}{|l|}{ Chest Radiography } \\
\hline \multicolumn{9}{|l|}{ Location in lung } \\
\hline Bilateral & $36(85.7)$ & $19(73.1)$ & $9(81.8)$ & $3(60.0)$ & $3(60.0)$ & $1(100.0)$ & $1(100.0)$ & $72(79.1)$ \\
\hline Simply left lung & $2(4.8)$ & $2(7.7)$ & $1(9.1)$ & $1(20.0)$ & $1(20.0)$ & $0(0.0)$ & $0(0.0)$ & $7(7.7)$ \\
\hline Simply right lung & $4(9.5)$ & $5(19.2)$ & $1(9.1)$ & $1(20.0)$ & $1(20.0)$ & $0(0.0)$ & $0(0.0)$ & $12(13.2)$ \\
\hline Diffuse lung (up and lower lobes) & $20(47.6)$ & $13(50.0)$ & $4(36.4)$ & $1(20.0)$ & $3(60.0)$ & $0(0.0)$ & $0(0.0)$ & $41(45.1)$ \\
\hline Infiltrates & $36(85.7)$ & $21(80.8)$ & $8(72.7)$ & $4(80.0)$ & $5(100)$ & $0(0.0)$ & $1(100.0)$ & 75 (82.4) \\
\hline Cavitation & $28(66.7)$ & $8(30.8)$ & $10(90.9)$ & $2(40.0)$ & $3(60.0)$ & $1(100.0)$ & $1(100.0)$ & $53(58.2)$ \\
\hline Nodules & $30(71.4)$ & $18(69.2)$ & $11(100)$ & $3(60.0)$ & $4(80.0)$ & $1(100.0)$ & $0(0.0)$ & $67(73.6)$ \\
\hline Miliary pattern & $0(0.0)$ & $0(0.0)$ & $0(0.0)$ & $0(0.0)$ & $0(0.0)$ & $0(0.0)$ & $0(0.0)$ & $0(0.0)$ \\
\hline Consolidation & $11(26.2)$ & $4(15.4)$ & $1(9.1)$ & $0(0.0)$ & $1(20.0)$ & $0(0.0)$ & $1(100.0)$ & $18(19.8)$ \\
\hline Pleural effusion & $4(9.5)$ & $5(19.2)$ & $0(0.0)$ & $0(0.0)$ & $2(40.0)$ & $0(0.0)$ & $1(100.0)$ & $12(13.2)$ \\
\hline Pleural thickness & $11(26.2)$ & $8(30.8)$ & $3(27.3)$ & $0(0.0)$ & $1(20.0)$ & $1(100.0)$ & $0(0.0)$ & $24(26.4)$ \\
\hline Adenopathy & $20(47.6)$ & $6(23.1)$ & $2(18.2)$ & $0(0.0)$ & $1(20.0)$ & $0(0.0)$ & $1(100.0)$ & $30(33.0)$ \\
\hline
\end{tabular}




\section{Competing interests}

The authors declare that they have no competing interests.

\section{Authors' contributions}

$\mathrm{HJ}$ and WT contribute equally to this work. XM, HJ, EG designed the study and wrote the manuscript; $\mathrm{HJ}$ and YD conducted laboratory experiments; WT collected patients' clinical data; DG, LL, ZL contributed to the study design and patients' diagnoses. All authors read and approved the final manuscript.

\section{Acknowledgments}

The authors thank Katharine Hsu Foundation for overall support of epidemiologic studies in Shandong. This work was supported by US NIH grant RO1 Al075465 and Shandong Taishan Scholarship (tshw20110537).

\section{Author details}

'Katharine Hsu International Research Center of Human Infectious Diseases, Shandong Provincial Chest Hospital, Shandong University, Jinan, Shandong 25013, China. ${ }^{2}$ Clinical Center on TB, Chinese Center for Disease Control and Prevention, Beijing 101149, China. ${ }^{3}$ Department of Laboratory Medicine, Shandong Provincial Hospital, Jinan, Shandong 250021, China. ${ }^{4}$ The Center for Molecular and Translational Human Infectious Diseases Research, Houston Methodist Hospital Research Institute, Houston, Texas 77030, USA.

Received: 4 June 2014 Accepted: 25 August 2014

Published: 11 September 2014

\section{References}

1. Shojaei H, Heidarieh P, Hashemi A, Feizabadi MM: Daei Naser A; Species identification of neglected nontuberculous mycobacteria in a developing country. Jpn J Infect Dis 2011, 64:265-271.

2. Griffith DE: Nontuberculous mycobacterial lung disease. Curr Opin Infect Dis 2010, 23:185-190.

3. Wang HX, Yue J, Han M, Yang JH, Gao RL, Jing L, Yang SS, Zhao YL: Nontuberculous mycobacteria: susceptibility pattern and prevalence rate in Shanghai from 2005 to 2008. Chin Med J 2010, 123:184-187.

4. Jing H, Wang H, Wang Y, Deng Y, Li X, Liu Z, Graviss EA, Ma X: Prevalence of Nontuberculous Mycobacteria Infection, China, 2004-2009. Emerg Infect Dis 2012, 18:527-528.

5. Griffith D, Aksamit T, Brown-Elliott B, Catanzaro A, Daley C, Gordin F, Holland SM, Horsburgh R, Huitt G, lademarco MF, Iseman M, Olivier K, Ruoss S, Von Reyn CF, Wallace RJ Jr, Winthrop K: An Official ATS/IDSA Statement: Diagnosis, Treatment, and Prevention of Nontuberculous Mycobacterial Diseases. Am J Respir Crit Care Med 2007, 175:367-416.

6. Kent PT, Kubica GP: Public Health Mycobacteriology: a Guide for the Level III Laboratory. Atlanta, GA: U.S. Department of Health and Human Services/ Public Health Service/Centers for Disease Control; 1985.

7. Han XY, Pham AS, Tarrand JJ, Sood PK, Luthra R: Rapid and accurate identification of mycobacteria by sequencing hypervariable regions of the $16 \mathrm{~S}$ ribosomal RNA gene. Am J Clin Pathol. 2002, 118:796-801.

8. Wong TC, Chan WF, Tsang WL, Yeung SH, Ip FK: Mycobacterium farcinogenes infection after total hip arthroplasty. J Arthroplasty 2005, 20:684-687.

9. Chinese Center for Disease Control and Prevention: The Fifth National Tuberculosis Surveillance of China. Beijing,China: Military Medical Science Press; 2011.

10. Adjemian J, Olivier KN, Prevots DR: Nontuberculous Mycobacteria among Cystic Fibrosis Patients in the United States: Screening Practices and Environmental Risk. Am J Respir Crit Care Med 2014 [Epub ahead of print].

doi:10.1186/2049-6958-9-48

Cite this article as: Jing et al:: Diagnostic delay of pulmonary nontuberculous mycobacterial infection in China. Multidisciplinary Respiratory Medicine 2014 9:48.

\section{Submit your next manuscript to BioMed Central and take full advantage of:}

- Convenient online submission

- Thorough peer review

- No space constraints or color figure charges

- Immediate publication on acceptance

- Inclusion in PubMed, CAS, Scopus and Google Scholar

- Research which is freely available for redistribution

Submit your manuscript at www.biomedcentral.com/submit
C Biomed Central 